\title{
Avanceurs de Phase
}

Le problème du factrur de paissance el des moyens de l'améliorer dans les installatons industrielles devienl chaque jour plus importanl. On a éludié différentes méthodes pour relever le facteur de puissance et améliorer ainsi les conditions de fonctionnement des installations, avec le maximum d'économie et le minimum de changement dans l'appareillage. Il n'y a pas de solution unique qui puisse s'appliquer indifféremment en raison du grand nombre de conditions que l'on rencontre dans les différentes installations.

Dans notre article sur les condensateurs synchrones (1), nous avons montré que l'emploi de ces machines permetlait d'améliorer facilement et économiquement le facteur de puissance d'une installation déjà existante. Cette solution est particulièrement recommandable aux industriets utilisant un grand nombre de petits moleurs d'induction, donl le contrat de fourniture d'énergie électrique impose un facteur de puissance minimum, el aux secteurs de distribution qui venlent améliorer le rendement de leur réseau et accroître leur puissance distribuée par une meilleure utilisation de leurs lignes el génératrices.

Dans la présente notice, nous allons examiner une autre solution du problème, qui s'applique plus spécialement aux installations comportant des moteurs d'induction de moyenne et de grande puissance. L'emploi anec ces moleurs d'un Avanceur de phase, qui permet de ramener pour ces unités le facteur de puissance à une valeur 'gule à l'unité, diminue ainsi dans une grande proportion la puissance réactive au réseau et améliore par ce fail même le facteur de puissance général de l’installation.

Historigut:

l'idée d'insérer dans les circuils roloriques d'un moteur d'induction des condensaleurs (ou des appareils dynamiques équivalents), en vue d'améliorer son cos ż, a élé émise el réalise par M. Maurice Leblane, en 1899, dans son appareil qu'il a appelé Récupérateur.

A la fin de 1901, se basant sur les proprićlés des anneaux collecteurs, mises en évidence par M. Marius l alour (2), il réalisa son Ananceur de phase, appelé encore couramment Compensateur de phase, sous la forme définilive d'une excitatrice spéciale composée d'un induil de machine à courant contimu muni d'un collecteur el alimenté au moven de balais par les courants rotoriques du moteur asynclrone à compenser. Le champ tournant, créé dans cel induit par les courants roloriques, se fermait au travers d'un stator ne portant aucun enroulement et dont le but élail de diminuer la réluctance du circuit magnétique.

La f. c. m. nécessaire pour la compensation étant relativement faible, on peut obtenir une commutation satisfaisante par une subdivision convenable du collecteur, d'autant plus qu'en l'absence d'entrefer, la saturation, pour ainsi dire inévitable, du circuit magnétique, vient limiter la f. e. m. engendrée à une valeur maximum encore compatible pour une bonne commutation.

Depuis, cette invention fut reprise et exploilée notamment à l'élranger par des auteurs qui ont cherché le plus souvent à s'altribuer le mérite de la découverte, et c'est pourquoi nous

(1) Voir la Houille Blanche de mai-juin 1923.

(2) Voir l'Eclairage Electrique, du 23 novembre 1901 , et la Lumière Electrique, du 12 juillet 1913. avons tenu à en préciser, très nellement, l'origine essentiellement française (1).

BLT DE L'ANANCELR DE PHASE.

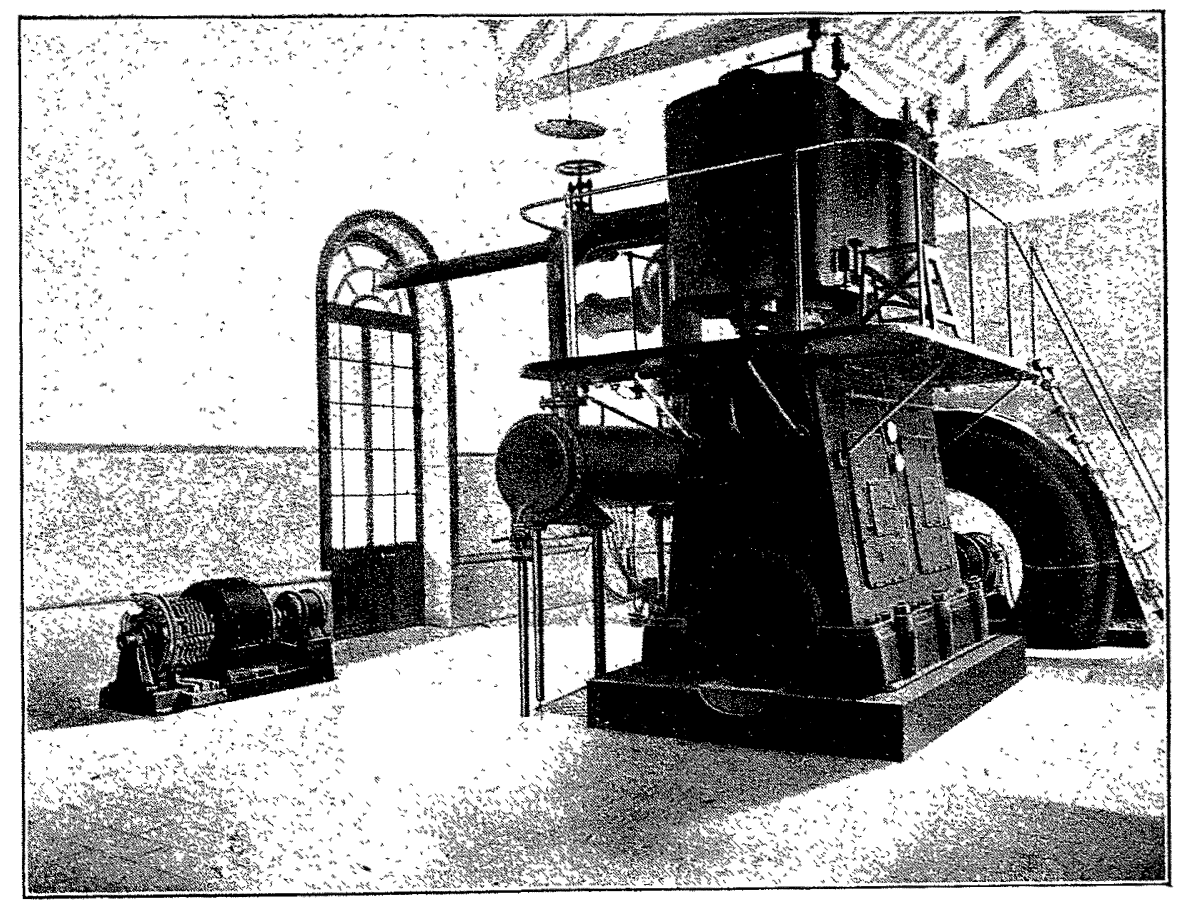

Fig. 1. - Vue d'un avanceur de phase adjoint à un moteur de $540 \mathrm{Ch} 187 \mathrm{t} / \mathrm{m}$ actionnant un compresseur d'air.

On sail que le courant absorbé sur un réseau par un moteur d'induction est la résultanle d'un courant en phase avec la tension d'alimentation, courant watlé dont la valeur correspond à la puissance développée par le moteur, el d'un courant décalé de $90^{\circ}$ en arrière sur la tension (courant déwatté ou magnétisant).

Le courant magnétisant à vide d'un moteur asy'nchrone est de l'ordre de 25 à $35 \%$ du courant de pleine charge. Ce courant qui ne produit aucune puissance augmente les pertes par effet Joule dans les enroulements et les lignes, tout en accentuant la baisse de tension sur le réseau.

Le but de l'avanceur de phase est de produire, dans chacun des circuits du rotor d'un moteur asynchrone, une force électromotrice de même périodicité que celle des courants rotoriques et décalée en avant de $\frac{\pi}{2}$ par rapport à ces mèmes courants. Pour une valeur convenable de cette f. e. m., on peut arriver à décaler en avant d'une valeur suffisante la f. e. $m$. résultante dans le rotor, de telle façon que la puissance magnétisante de ce dernier soit égale ou même supérieure à celle du stator et parvienne ainsi à annuler le courant magnétisant pris au réseau.

Les modifications qu'apporte cette f. e. m. injectée dans les

(1) Voir The Electrician, du 16 mai 1913, lettre de M. Gratzmuller. 
circuits rotoriques se déterminent facilement en se reportant au diagramme classique du cercle.

Représentons fig. 2 par O A la somme des ampères tours magnétisants du slator produisant le flux $\Phi$ dans la marche à vide.

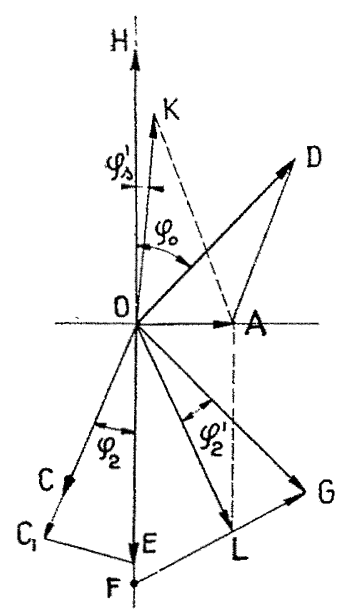

FIG. 2.
On sait que dans un moteur asyuchrone ordinaire les ampèrestours produisant le flux sont la résultanle de la somme des ampères-tours du stator représentée en $O D$ et de la somme des ampères-tours du rotor représentée en $\mathrm{O} C$, cette résultante étant égale d'ailleurs aux ampèrestours du stator dans la marche à vide.

Les ampères-tours du rotor sont déterminés, d'une part, par le couple à vaincre qui est proportionnel au produit du flux \$ par la composante wattée du courant rotorique $\mathrm{I}_{2}$, par le nombre $\mathrm{N}$ des conducteurs du rotor, et, d'autre part, par le décalage $\vartheta_{2}$ du courant rotorique sur la f. e. $m . E_{\mathrm{rg}}$ du rotor défini par la relation :

$$
\operatorname{tg} p_{2}=\frac{\mathrm{L}_{\mathrm{z}} \cdot \omega_{\mathrm{g}}}{\mathrm{R}_{\mathrm{2}}}
$$

avec $\omega_{\mathrm{g}}=g \Omega, g$ étant le glissement, $\Omega$ la pulsation des courants du stator, $L_{2}$ et $R_{2}$ étant respectivement le coefficient de self-induction dû aux fuites et la résistance d'une phase du rotor.

Sur le diagramme, le vecteur $\mathrm{O} E$ représente la f. e. $m$. $\mathrm{E}_{\mathrm{rg}}$ développée dans le rotor sous l'influence du glissement $g$ et décalée de $\overline{\frac{\pi}{2}}$ en arrière sur le flux résultant, le vecteur diamétralement opposé O H représente la tension d'alimentation du stator formant avec le courant $O D$ l'angle $c_{0}$.

Le vecteur $O E=E_{r g}$ est la résultante du vecteur $O C_{1}$ représentant la chute ohmique $\mathrm{R}_{2} \mathrm{I}_{2}$ dans une phase du rotor et du vecteur $\mathrm{C}_{1} \mathrm{E}$ perpendiculaire au premier, représentant la chute due à la self-induction $\omega_{\mathrm{g}} \mathrm{L}_{2} \mathrm{I}_{2}$.

Injectons maintenant dans chaque phase du rotor une f. e. $m$. $F \mathrm{G}$ de sens convenable et de même fréquence que les courants rotoriques; nous obtenons en $\mathrm{O} G$ la f. e. $\mathrm{m}$. résultánte dans le rotor qui produira un courant décalé en arrière d'un angle $\rho_{i}^{\prime}$, tel que :

$$
\operatorname{tg} p_{2}^{\prime}=\frac{L_{2}^{\prime} \cdot \omega_{g}^{\prime}}{R_{2}^{\prime}}
$$

$L_{2}, \omega_{g}, R_{2}^{\prime}$ étant les nouvelles valeurs du coefficient de selfinduction, du glissement et de la résistance dans le rotor, après l'adjonction de l'avanceur de phase.

Le vecteur $\mathrm{O} H$ représentera maintenant les ampères-tours du rotor qui ,composé avec $\mathrm{O} \mathrm{A}$, donnera le vecteur $\mathrm{O} \mathrm{K}$, représentant les nouveaux ampères-tours du stator décalés de ?'s sur la tension U's.

On voit par ce qui précède que, suivanl la grandeur de la f. e. m. injeclée $E_{c}=F G$, on peut ramener le facteur de puissance du stator à telle valeur que l'on désire et même décaler en avant le courant statorique.

\section{Principe du Fonctionnement d'Un AVANGEUR de PHASE.}

Le principe de l'avanceur de phase découle immédiatement des propriétés d'un induit à collecteur muni de balais et tournant dans un champ inducteur mobile (1).

(1) Pour l'étude plus complète, nous renvoyons le lecteur aux ouvrages techniques, notamment au cours d'Electrotechnique appliquée, de M. Mauduit, page 994 et suivantes (édition 1922).
Les quelques considéralions suivantes, dans lesquelles nous n’avons point l'intention de faire des démonstrations absolument rigoureuses, permeltront néanmoins au lecteur de comprendre aisément le principe du fonctionnement.

Considérons tout d'abord un induit bipolaire de dynamo à courant continu, placé dans le champ magnétique des pôles $\mathrm{N}$ et S (fig. 3).



On sait que si les balais sont placés dans la ligne neutre, la différence de potentiel $B_{1} B_{2}$ est égale à

$$
\mathrm{E}_{\mathrm{c}}=\frac{\mathrm{N} \cdot n \cdot \Phi}{60 \times 10^{8}}
$$

dans laquelle $\mathrm{N}$ est le nombre de tours de l'induit par minute, $n$ le nombre de conducteurs, $\Phi$ le flux qui traverse l'induit.

Comme $\frac{N}{60}=\frac{\omega}{2 \pi}, \omega$ étant la vitesse angulaire de l'induit

$$
\mathrm{E}_{\mathrm{c}}=\frac{n \Phi}{2 \pi 10^{8} \omega}
$$

On sait également que si les balais, au lieu d'être placés sur la ligne neutre, c'est-à-dire décalés de $\frac{\pi}{2}$ par rapport à l'axe origine $\mathrm{O} X$, sont décalés d'un angle $\alpha$, la différence de potentiel entre balais devient :

$$
\mathrm{U}=\mathrm{E}_{\mathrm{c}} \sin \alpha
$$

Si l'on suppose les inducteurs animés d'un mouvement de rotation de vitesse angulaire $\Omega$ et dans le même sens que l'induit, l'angle $\alpha$ séparant l'axe des pôles de l'axe des balais maintenus fixes sera donné par :

$$
\alpha=\Omega t
$$

par un choix convenable de l'origine du temps.

Dans ces conditions, la différence de potentiel entre balais varie périodiquement et devient

$$
\mathrm{U}=\mathrm{E}_{\mathrm{c}} \sin \alpha=\mathrm{E}_{\mathrm{c}} \sin \Omega t
$$

Par contre, la vitesse relative de l'induit par rapport aux inducteurs est égale à $(1)-\Omega$, et se trouve réduite dans le rapport $\frac{\Omega}{0}$; par conséquent, la f. e. m. induite est diminuée dans le mème rapport et devient :

$$
\mathrm{E}_{(\Omega)}=\mathrm{E}_{\mathrm{c}}\left(\mathrm{I}-\frac{(\omega)}{\Omega}\right)=\mathrm{E}_{\mathrm{c}}\left(\frac{(\omega)-\Omega}{\omega}\right)
$$

Par suite, la différence de polentiel entre les balais fixes devient, d'après la formule (2) :

$$
\mathrm{U}=\mathrm{E}_{\mathrm{c}} \frac{(\omega)-\Omega}{\omega} \sin \Omega t
$$

Nous voyons finalement que la différence de potentiel entre 
deux balais placés diamétralement sur le collecteur d'un induit bipolaire, tournant dans le même sens que les inducteurs, est sinusoüdale de pulsation égale à celle du champ inducteur, el indépendante de la vilesse de rotation de l'induit.

La rotation du champ inducleur peut s'obtenir très simplement en employant un stator polyphasé alimenté par des courants polyphasés. En particulier, si ce stator est alimenté par les courants rotoriques d'un moteur asynchrone polyphasé, nous pourrons recueillir au collecteur une f. e. m. de même périodicité que ces courants rotoriques et de même nombre de phases si l'on dispose sur le collecteur des lignes de balais espacées convenablement.

Nous réalisons ainsi la première condition que doit remplir un avanceur de phase.

Il nous reste à démontrer maintenant que cette différence de potentiel est décalée de $\frac{\pi}{2}$ sur le courant inducteur.

Pour permeltre au lecteur de suivre plus facilement, nous considérerons un compensateur bipolaire diphasé avec enroulement en anneau (fig. 4). Le collecteur comporte quatre lignes de balais placées sous l'axe des pôles, et nous supposons que le sens de rolation du champ tournant inducteur est le même que celui de l'induit.

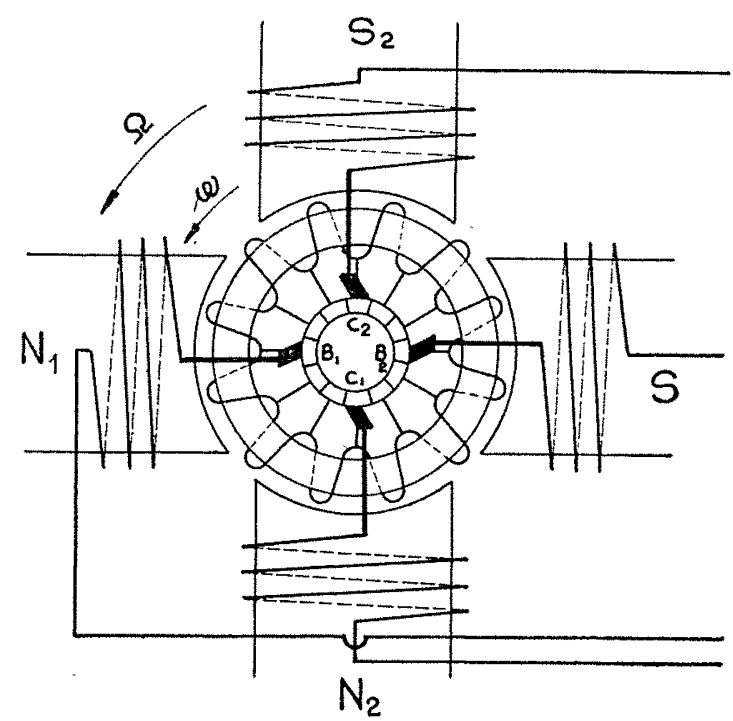

FIG. 4.

Dans un système diphasé, on sait qu'à l'instant où le courant $\mathrm{I}_{1}$ de la phase 1 est maximum, le courant $I_{2}$ de la phase 2 est nul; les pôles $N_{1} S_{1}$ alimentés par le courant $I_{1}$ seront donc seuls excités à cet instant, et la différence de potentiel entre balais $B_{1} B_{2}$ faisant partie du circuit de la phase i sera nulle, alors qu'elle est maximum entre les balais $\mathrm{C}_{1} \mathrm{C}_{2}$ insérés dans la phase 2 .

$A$ l'instant où le courant $I_{2}$ sera maximum, c'est-à-dire lorsque le champ inducteur aura tourné de $\frac{\pi}{2}$, la différence de potentiel devient nulle entre les balais $\mathrm{C}_{\mathrm{C}_{2}}$ et maximum entre les balais $\mathrm{BB}_{2}$.

Ainsi donc la f. e. m. recucillie et le courant qui traverse'les balais sont en quadrature, ces derniers étant placés sous l'axe des pòles.

Nous voyons, en outre, sur la fig. 4 que les courants se répartissent dans l'induit d'une façon symétrique par rapport aux pôles excités, de sorte que le couple résultant est nul. De plus, le champ inducteur et le champ propre de l'induit sont concordants, on peut donc utiliser cette propriété pour supprimer les enroulements inducteurs en renforçant le champ induit.

Si nous nous reportons à la formule (4) qui nous donne la différence de potentiel entre balais

$$
\mathrm{U}=\mathrm{E}_{\mathrm{c}} \frac{\omega-\Omega}{(1)} \sin (\Omega t)
$$

nous voyons que le signe de cette différence de potentiel dépend des valeurs respectives de (1) et (2. En particulier, lorsque l'induit est immobile $(\omega=0)$, l'avanceur de phase se comporte comme une self placée dans le circuit du rotor du moteur asynchrone et le courant est décalé de $\frac{\pi}{2}$ en arrière sur la tension aux balais, en négligeant la résistance des circuits de l'avanceur.

Pour $\omega=\Omega$ la f. e. m. engendrée par l'avanceur devient nulle et ce dernier se comporte comme une simple résistance.

Enfin, pour $\omega>\Omega$ la f. e. m. change de signe et le courant qui était décalé de $\frac{\pi}{2}$ en arrière dans le premier cas par rapport à la tension se trouve maintenant décalé de $\frac{\pi}{2}$ en avant.

Pour un avanceur de phase triphasé le cas est un peu plus compliqué, du fait que l'induit forme un système en triangle, alors que les conducteurs aboutissant aux balais forment un système en étoile. Il faut, dans ce cas, comparer la différence de potentiel $\mathrm{V}_{\mathrm{B} 1}-\mathrm{V}_{\mathrm{B} \hat{2}}$ au courant résultant $\overline{\mathrm{I}}_{1}-\mathrm{I}_{2}$ en avance de $30^{\circ}$ sur le courant $I_{1}$.

Il y a encore lieu de noter que dans ce cas, la différence de potentiel entre balais n'est que les 0,866 de la force électromotrice maximum induite.

Modifications apportées par un avanceur de phase dans Le FONCTIONNEMENT D'UN MOTEUR ASYNCHRONE.

Nous avons vu par le diagramme de la fig. 2 comment se modifie le facteur de puissance d'un moteur asynchrone, lorsqu'on introduit dans le rotor une f. e. $\mathrm{m}_{\mathrm{c}} \mathrm{E}_{\mathrm{c}}$ de grandeur donnée, produite par un avanceur.

Rappelons que cette f. e. m. a pour valeur en triphasé,

$$
\mathrm{E}_{\mathrm{a}}=0,866 \frac{\Phi}{10^{8}} \frac{\mathrm{N}}{2 \pi}(\omega-\Omega) \sin \Omega t
$$

ou, en valeur efficace,

$$
\mathrm{E}_{\mathrm{a}} \text { eff. }=\frac{0,866}{\sqrt{2}} \frac{\Phi}{10^{8}} \frac{\mathrm{N}}{2 \pi}(10-\Omega)
$$

Le flux ' étant produit par l'enroulement induit est fonction du courant et sera donné par la caractéristique à vide de l'avanceur.

La vitesse angulaire $(\omega-\Omega)$ dépend d'une part de la vitesse du moteur d'entraînement et, d'autre part, de la fréquence des courants rotoriques du moteur principal.

On peut donc calculer en grandeur la f. e. m. E pour chaque valeur du courant rotorique.

Les modifications qui résultent de l'introduction de cette f. e. m. dans le diagramme d'un moleur asynchrone sont assez complexes, et leur étude complète sortirait du cadre de cette notice.

Nous domnons ci-dessous (fig. 5) le diagramme modifié pour un moteur asynchrone de $1.000 \mathrm{Ch}-5.000 \mathrm{~V}$. $-250 \mathrm{t} / \mathrm{m}$, muni d'un avanceur de phase de $11 \mathrm{kVA}$.

La comparaison avec le diagramme du moteur sans avanceur de phase permettra au lecteur de saisir plus facilement les principales différences qui résultent du fonctionnement avec avanceur.

Remarquons que le glissement se trouve un peu augmenté du fait de l'adjonction de l'avanceur de phase, notamment aux faibles charges, mais ce glissement ne représente plus les pertes rotoriques.

Nous voyons également que le couple maximum du moteur se trouve augmenté. 
Ajoutons que ces appareils peuvent aussi etre calculés pour produire un décalage en avant du courant du stator et fournir ainsi une partie du courant réactif nécessaire à l'excitalion des pelits moleurs qui composent le reste de l'installation. Dans ce eas, le rotor du moteur doit être dimensionné plus largement pour éviter l'échauffement. Celte dernière solution conduit d'ailleurs à un avanceur de phase puissant, partant coûteux, et n'est pas à rechercher.



Nota. - Lorigine n a cté reportée en o'.

Fic. 5. - Diagramme de fonctionnement avec ou sans avance ur de phase d'un moteur asynchrome triphase de 1000 (h. jo00 voll.

Pratiquement, le cos o du moleur compensé est sensiblement égal à 1 depuis le $1 / 4$ de charge jusqu'aux $5 / 4$ de charge.

L'avanceur de phase convient particulièrement aux moleurs asynchrones à faible vilesse où, pour des raisons de stabilité, on est conduil à de faibles cos ₹ et à de pelits entrefers. Avec un avanceur on peut ainsi consentir à des entrefers plus grands et à une stabilité meilleure.

\section{Description DE L'AVANCEUR DE phase.}

La théorie que nous avons exposée dans les pages précédentes sur le fonclionnement d'un avanceur de phase nous a déjà permis d'indiquer les éléments constitutifs de cette machine.

La description plus détaillée que nous donnons ci-dessous se rapporte à un type courant d'avanceur de phase dont le stator non bobiné fait corps avec le rotor. L'examen des photographies et le plan d'ensemble de la fig. 6, se rapportant à cet appareil, montrent le dimensionnement des"divers organes.

La partie principale est constituée par un induit sensiblement analogue à un induil de machine à courant conlinu qui serail prévu pour une tension relativement faible el une grande inlensité.

Le noyau de l'induit, qui constilue en même lemps le stator, est constilué par des tôles circulaires en acier doux à faibles pertes, montées sur un croisillon fixé lui-même sur l'arbre; ces tôles comportent des encoches complètement fermées dans lesquelles sont logés les conducteurs convenablement isolés.

Lc bobinage ne présente pas de particularilés essentielles. Il est identique à l'enroulement imbriqué d'un induit en tambour' d'une machine à courant continu ayant le mème nombre de pôles. Il est protégé par un capot démontable. L'enroulement d'excilation est constitué par l'enroulement induil lui-même, car, ainsi qu'il a élé dit, flux induit et flux inducleur sont de même sens dans le foncliomnement normal de ces appareils.

Le collecteur est l'organe principal. Il est dimensionné pour supporter sans échauffement les courants rotoriques. Sa longueur est de ce fait prévue en conséquence. Les lignes de balais sont d'un accès facile pour l'entretien el le changement éventuel des charbons ; le collecteur comporte trois rangées de balais par double pas polaire dans le cas du triphasé, les lignes de balais de même phase étant réunies en parallèle.

Le sens de rolation du flux tournant dans l'induil dépend de l'ordre des connexions aux balais el la vilesse angulaire de ce champ tournant est indépendante de la vilesse de l'induit par suite du collecteur, lout comme dans une machine à courant contimu.

L'arbre est monté sur deux paliers séparés, fixés eux-mèmes sur un socle en fonte ainsi que le petit moteur d'entrainement de l'avanceur de phase. Ia puissance de ce dernier est relativement très laible el correspond uniquement aux perles mécanique's dans l'avanceur. Nous avons vu, cn effel, que le couple ulile dans celle machine est nul.

Jes avanceurs de phase peuvent encore se construire avec un stator indépendant, séparé du rotor par un entrefer el ne comportant, comme dans le cas précédent, aucun bobinage. Il est conslitué dans ce cas par un paquel de lòles circulaires concentriques au rolor. Celle disposition, si elle offre des avantages de cons" truction, présente des inconvénients au point de vue commulalion dans le cas de forles surcharges du moteur principal.

Enlin, le stator des avanceurs de phase, destiné à compenser de gros moteurs, peut recevoir un bobinage alimenté également par les courants rotoriques. Il peul être construil à pôles saillants ou à surface continue. Du fail que la commulation s'opère sous l'axe des pôles, on munit le stator d'enroulements de compensation el de commutation par une répartition convenable des connexions entre les différents circuits.

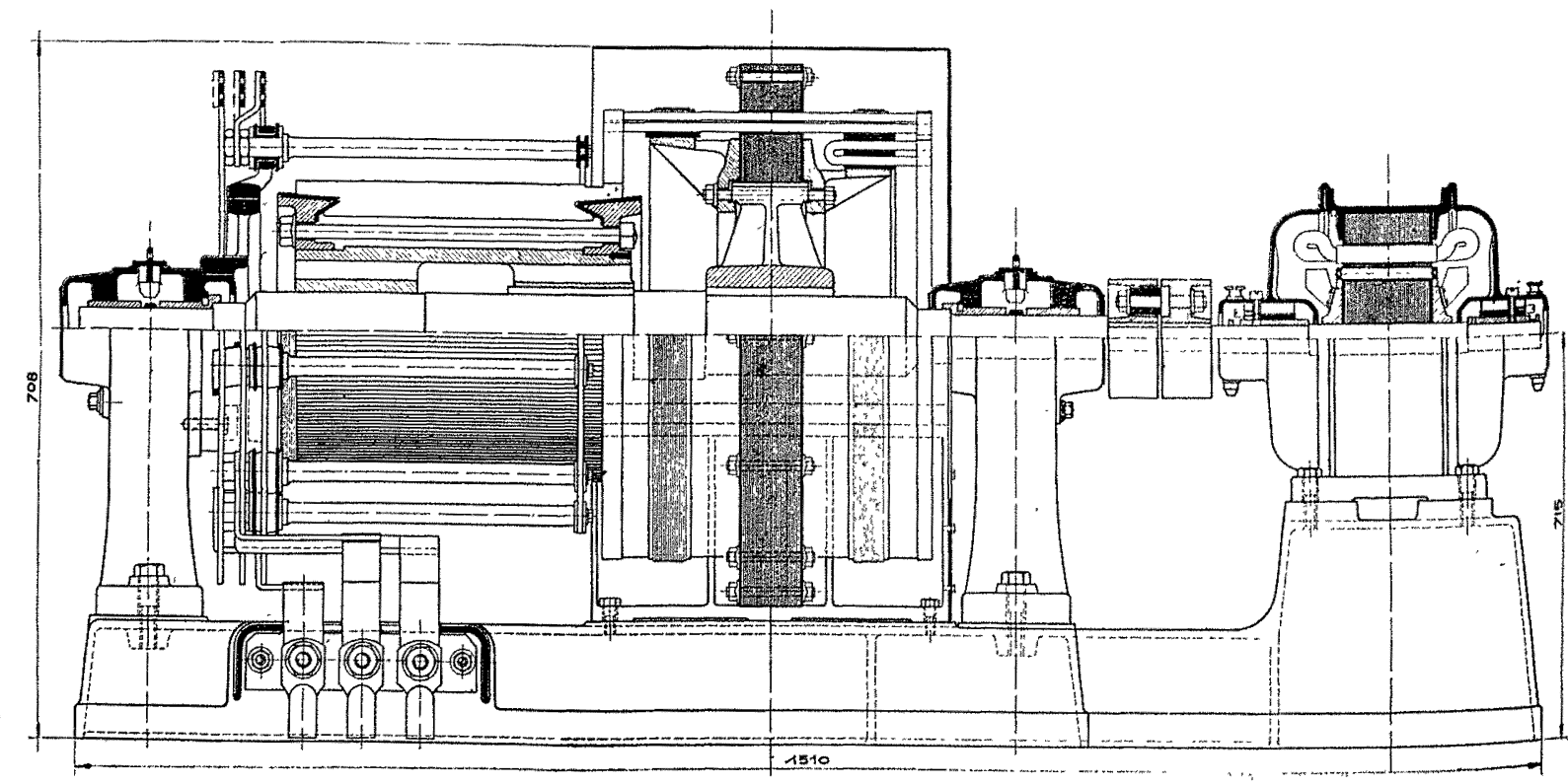

Fig. 6. - Plan d'ensemble d'un avanceur de phase, 
INSTALLATION LET SCHÉMA DE MONTAGE D'UN AVANCLUR DE PIASE

Le schéma de l'installation doil être prévu pour permeltre la mise hors circuil de l'avanceur de phase pendant la période de démarrage. Ce dernier s'effectue en insérant, au moyen d'un rhéostal des résistances convenables dans les phases du rotor. De ce fait, le moteur asynchrone conserve au point de vue démarrage loules ses propriélés.

Si la mise en circuil avail lieu au moment du démarrage, l'avanceur de phase pourrait être délérioré par la haute tension de lenroulement secondaire.

Les disposilifs prévus sur le rhéostat de démarrage permeltenl de brancher l'avanceur de phase sur les bagues du rotor, lorsque la vitesse de ce dernier a alleint sa valeur normale, et de meltre en même temps le rhéostat hors circuil. Un dispositif de verrouillage flectrique est généralemenl prévu pour empecher loule fausse manceuvre.

le schéma de la fig. 7 donne l'enlion d'un moteur asynchrone de 510 (h $5.500 \mathrm{~V},--187 \mathrm{l} / \mathrm{m}$ muni d'un avanceur de phase. semble des commexions pour l'installa-



2. Avinceur de phase.

4 Rhéostat liquide. vanceur. de circuit. $6001 \% / 110 \mathrm{Y}$.

13 Boites d'extrimité.

15. Voltmelre. rapteur.

18. Lampes signaux.
Fig. 7. - Schéma des comnexions d'un moleur de 510 Ch. avec avanceur de phase.

LEGENDE

1. Noteur de 540 Ch. -5700 V. $-550 \mathrm{pps}$.

3. Moteur de lavanceur.

5. Comrnutateur de commande de la-

6. Sectionneurs $6000 \mathrm{~V},-100 \mathrm{~A}$

7. Coupe circuits $0000 \mathrm{r}$.

. Inverseur bipolaire pour la marche avec et sans avanceur

. Relais bipolaires à maš et ouverture

10. Transformateur de poten.iel mon.

11 Transformateur d'intensite $75 / 10 \mathrm{~A}$

19 Interrupteur tripolaire dans thuile.

If Ampéremètre électromannéliqus.

16. Bohine pour déclanchement maxima.

Bobine de verrouillage de Minter-

ApPlications GT AVANTAGES RÉSILTANT DE L'EMPLOI D'UN ANANCLAR DE PHASE.

Nous domnons ('i-dessous :

1.- I.es résullats des essais effectués par le service électrique de l' "Association Lyonnaise des Propriélaires d'Appareils à Vapeur "sur un moteur asynchrone triphase : $540 \mathrm{Ch}-187 \mathrm{l} / \mathrm{m}-$ $5.700 \mathrm{~V} .-50 \mathrm{pps}$, actionnant un compresseur d'air el construit ainsi que l'avanceur de phase par les " Ateliers de Constructions Electriques de Lyon et du Dauphiné" pour la Société Anonyme des Mines de la Loire à Saint-Etienne.

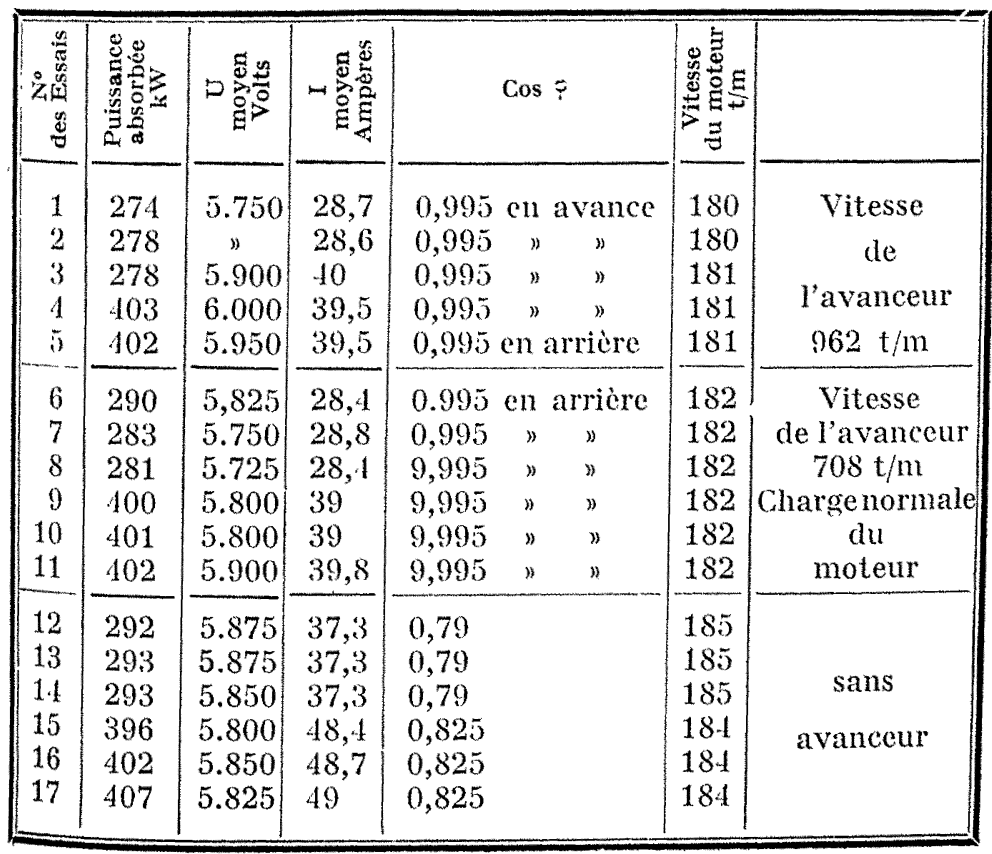

\begin{tabular}{|c|c|c|c|c|}
\hline 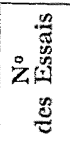 & 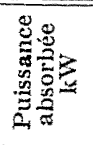 & - & ט. & OBSERVATIONS \\
\hline & & & & $\begin{array}{c}\text { Fonctionnement avec avanceur de phase } \\
\text { sous la tension de } 5.000 V \text {. mainlenue } \\
\text { constante. }\end{array}$ \\
\hline 1 & 735 & 82 & 1 & ¿ presses en marche. \\
\hline 2 & 611 & 65 & 1 & - \\
\hline 3 & 763 & 85 & 1 & Glissement $2,350$. \\
\hline 4 & 783 & 90,5 & 1 & 6 presses en marche. \\
\hline 5 & 875 & 103 & 1 & - \\
\hline 6 & 650 & 75 & 1 & $5-$ \\
\hline 7 & 611 & 70 & 1 & 4 presses en marche. Glissement $2 \%$ \\
\hline $\begin{array}{l}8 \\
9\end{array}$ & 510 & 57 & 1 & $\begin{array}{l}4 \\
4\end{array}-$ \\
\hline $\begin{array}{r}9 \\
10\end{array}$ & $\begin{array}{l}550 \\
476\end{array}$ & $\begin{array}{c}62,5 \\
54\end{array}$ & $\begin{array}{l}1 \\
1\end{array}$ & $\begin{array}{l}4 \\
3 \text { presses en marche. }\end{array}$ \\
\hline 11 & 494 & 56,5 & 1 & 3 presses en marche. \\
\hline 12 & 331 & 37 & 1 & 2 presses en marche. \\
\hline 13 & 298 & 34 & 0,95 & $2-$ \\
\hline 14 & 291 & 34 & 0,95 & $2 \quad-$ \\
\hline 15 & 80 & & $\begin{array}{r}0,40 \\
\text { e nviron }\end{array}$ & $\begin{array}{l}\text { Marche à vide des deux défibreurs. } \\
\text { Glissement } 0,75 \% \text {. }\end{array}$ \\
\hline 16 & 745 & 98 & 0,85 & $\begin{array}{l}\text { Fonctionnement sans avanceur. } \\
5 \text { presses en marche. }\end{array}$ \\
\hline 17 & 790 & 103 & 0,85 & $5 \quad-$ \\
\hline 18 & 840 & 111 & 0,855 & - \\
\hline 19 & 634 & 88,5 & 0,835 & 4 presses en marche. \\
\hline 20 & 650 & 89,5 & 0,845 & - \\
\hline 21 & 431 & 64,5 & 0,77 & 3 presses en marche. \\
\hline 22 & 400 & 59 & 0,75 & $3-$ \\
\hline 23 & 79,5 & 33 & 0,28 & Marche à vide des deux défibreurs. \\
\hline
\end{tabular}


Les graphiques ei-joints montrent suffisamment les avantages que l'on peut retirer de l'emploi d'un avanceur de phase.

D'après ces essais, le moteur de $540 \mathrm{Ch}$ prend au réseau comme puissance magnétisante, à sa charge normale :

Sans avanceur ............. $275 \mathrm{kVA}$ Avec avanceur à sa vitesse normale. $40 \mathrm{kVA}$

soit une différence en moins de.... $235 \mathrm{kVA}$ et le moteur de $1.000 \mathrm{Ch}$ absorbe comme puissance dẹwattée à sa charge normale :

Sans avanceur............. $400 \mathrm{kVA}$ Avec avanceur, sensiblement......

Par cette pui.sance magnétisante rendue en quelque sorte disponible, on voit l'avantage que peuvent retirer les Industriels et les réseaux en munissant d'avanceurs de phase les moteurs de moyenne et de grande puissance.

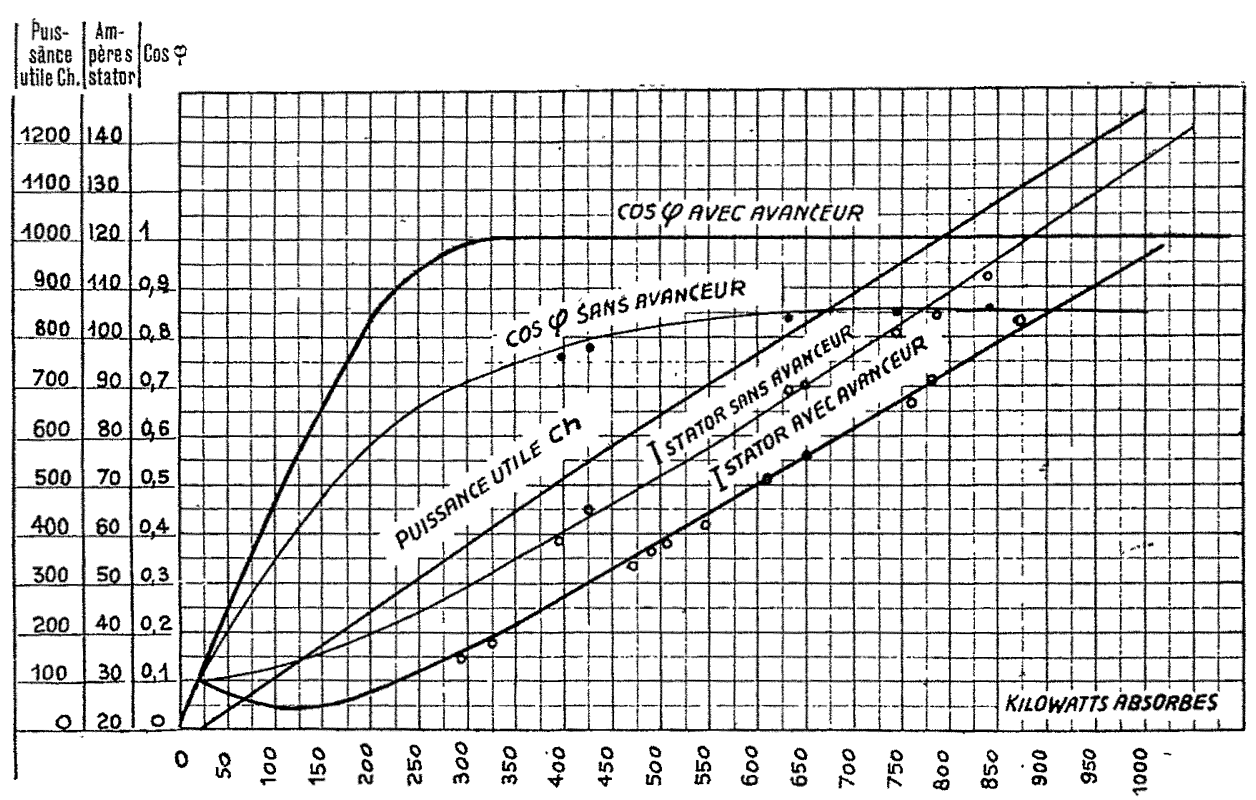

FIG. '9. - Diagramme des essais d'un moteur asynchrone triphasé de 1000 Ch. $250 \mathrm{t} / \mathrm{m} 5000 \mathrm{~V}$. $50 \mathrm{pps}$. avec et sans avanceur de phase.

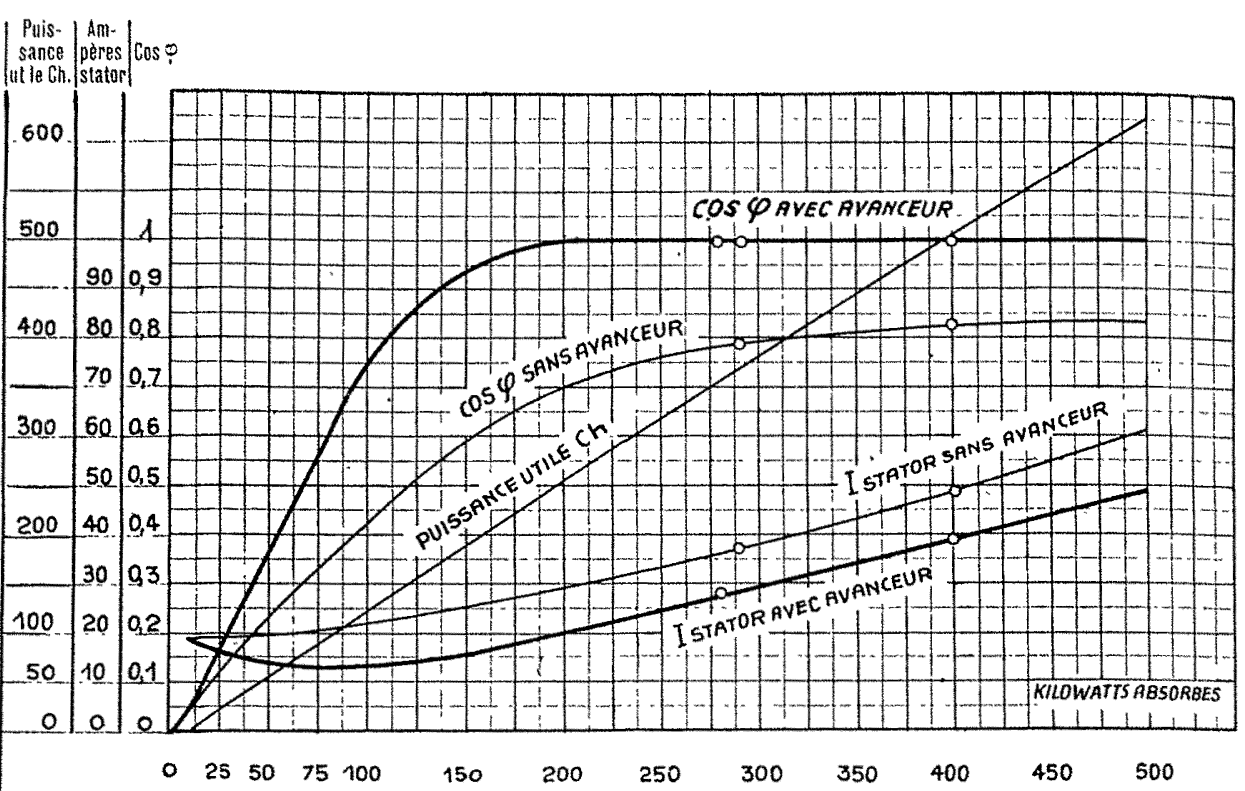

FIG. 8. - Diagramme des essais d'un moteurasynchrone triphaséde 540 Ch. $187 \mathrm{t} / \mathrm{m} 5700$ V. 50 pps. avecet sans avanceur de phase.

En prenant comme exemple un réseau dis* tribuant $500 \mathrm{Ch}$ de force en petits moteurs sous un cos 9 moyen de 0,7 environ, et $500 \mathrm{Ch}$ de force répartis en quelques moteurs de 100 à 150 Ch sous un $\cos$ p moyen de 0,82 , le $\cos \rho \mathrm{du}$ réseau serait de 0,76 , correspondant à une puissance magnétisante de $480 \mathrm{kVA}$.

En adjoignant à ces derniers moteurs des avanceurs de phase relevant leur cosinus à l'unité, sensiblement, le $\cos$ o du réseau remonterait à 0,92 , correspondant à une nouvelle puissance magnétisante de $260 \mathrm{kVA}$, d'ou un grain de $220 \mathrm{kVA}$ en puissance déwattée se traduisant par une diminution de $10 \%$ du courant débité.

L'avanceur de phase peut être adjoint à tout moteur existant, et son emploi est tout indiqué pour les moteurs à vitesse lente et pour ceux de grande et de moyenne puissance. Il offre l'avantage de créer le courant réactif au point où il est nécessaire et d'améliorer corrélativement la stabilité du moteur.

(Extrait du Bulletin 1009 des Ateliers de Constructions électriques de Lyon et du Dauphiné).

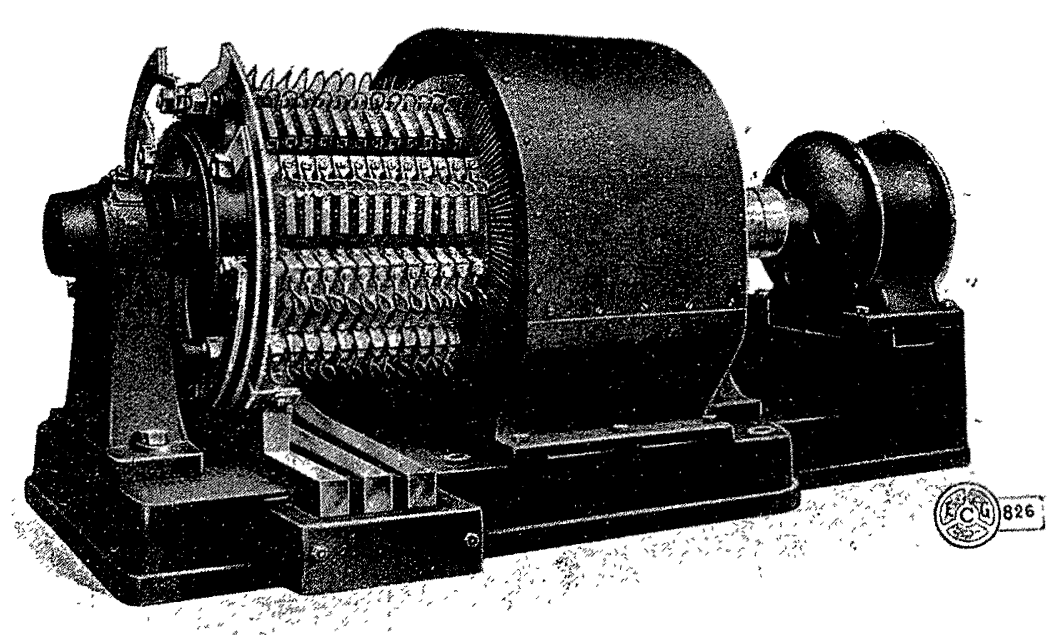

